\title{
Potencialidades Farmacológicas da Babosa: um estudo realizado por meio das técnicas de prospecção científica e tecnológica
}

\author{
Pharmacological Potentials of Babosa: a study carried out by scientific \\ and technological prospection techniques
}

\author{
Rayane Amorim Carvalho ${ }^{1}$ \\ Antonio Marcos da Conceição Lima ${ }^{1}$ \\ Álvaro Itáuna Schalcher Pereira ${ }^{1}$ \\ Oswaldo Palma Lopes Sobrinho ${ }^{2}$ \\ Francisco Adelton Alves Ribeiro ${ }^{1}$ \\ Sara Tamiris da Silva Costa ${ }^{3}$ \\ Terezinha Yasmin Azevedo Lopes ${ }^{4}$ \\ ${ }^{1}$ Instituto Federal de Educação, Ciência e Tecnologia do Maranhão, Codó, MA, Brasil \\ ${ }^{2}$ Instituto Federal de Educação, Ciência e Tecnologia Goiano, Rio Verde, GO, Brasil \\ ${ }^{3}$ Instituto Educacional Santa Catarina, Faculdade Jangada Instituto Nacional de Cursos, Teresina, PI, Brasil \\ ${ }^{4}$ Universidade Brasil, Codó, MA, Brasil
}

\begin{abstract}
Resumo
Este estudo realizou o monitoramento científico e tecnológico da Babosa com o intuito de fazer um mapeamento de seu progresso no campo científico e tecnológico. Para tanto, foram empregadas técnicas de prospecção científica e tecnológica, usando as bases de dados Scopus e World Intellectual Property Organization (WIPO) como banco de dados. Constatou-se que a Índia é detentora do maior número de publicações de artigos científicos, enquanto os Estados Unidos foi o país responsável pelo maior número de patentes depositadas envolvendo essa espécie. O Brasil apresentou baixa produção científica e tecnológica quando comparado a outros países. Quanto à CIP, a subclasse mais categorizada foi a A61K, apontando para o maior uso da Aloe vera no desenvolvimento de produtos com finalidades médicas e odontológicas.
\end{abstract}

Palavras-chave: Aloe vera. Artigos científicos. Patentes.

\begin{abstract}
The objective was to carry out the scientific and technological monitoring of this species in order to map its progress in the scientific and technological field. To this end, scientific and technological prospecting techniques were employed, using the Scopus and World Intellectual Property Organization (WIPO) databases as databases. It has been found that India has the largest number of publications of scientific articles, while the United States was responsible for the largest number of patents filed involving the aloe species. Brazil had low scientific and technological production when compared to other countries. Regarding CIP, the most categorized subclass was the A61K, pointing to the greater use of Aloe vera in the development of products for medical and dental purposes.
\end{abstract}

Keywords: Aloe vera. Scientific articles. Patents.

Área Tecnológica: Prospecções Tecnológicas de Assuntos Específicos. 


\section{Introdução}

A espécie Aloe vera é conhecida no Egito antigo como a "planta da imortalidade", ela teria sido usada por Cleópatra nos cuidados da pele e do cabelo (FREITAS; RODRIGUES; GASPI, 2014). É uma planta que se desenvolve com relação ao seu crescimento naturalmente em climas secos e quentes. Em sua composição, é constituída pelo tecido chamado mucilagem, que é composto aproximadamente de $98 \%$ de água e de $2 \%$ de outros compostos 75 bioativos (flavonoides, saponinas, esteroides, aminoácidos, sais minerais, vitaminas, etc.), cujas concentrações podem variar de acordo com as espécies, assim como com as condições de crescimento e clima (PARENTE et al., 2013).

Assim, diversas são as atividades biológicas evidenciadas pela Aloe vera, entre as quais podem ser citadas as atividades de conservação de alimentos, de clareamento da pele, potencial antimicrobiano, imunomoduladora, hepatoprotetora, hipoglicemiante, antitumoral, antioxidante, anti-inflamatória, nefroprotetora, cicatrizante de feridas e de queimaduras (BACH; LOPES, 2007; PATROCÍNIO; MACILHIA, 2012). Inclusive seu uso também é reportado na grande área das Ciências Agrárias.

Os estudos de prospecção são importantes, porém ainda são pouco explorados no Brasil. Nesse aspecto, conforme apontam Pereira et al. (2015), as bases de artigos científicos e patentes vêm se apresentando como ricas fontes de informações científicas e tecnológicas. Elas podem ser acessadas a todo momento de qualquer parte do mundo, o que facilita na recuperação dos dados. Diante do exposto, tem-se como objetivo realizar uma prospecção científica e tecnológica relacionada à espécie Aloe vera nas bases de dados Scopus e WIPO a fim de mapear as produções científicas e tecnológicas.

\section{Metodologia}

O presente trabalho é um estudo de caso, pois consiste no estudo profundo e exaustivo de um objeto de estudo, de maneira que permita seu amplo e detalhado conhecimento (GIL, 2002). Trata-se de uma pesquisa descritiva, pois tem como objetivo primordial a descrição das características de determinado fenômeno e o estabelecimento de relações entre variáveis existentes. E também se classifica como explicativa, uma vez que busca identificar os fatores que determinam ou que contribuem para a ocorrência dos fenômenos.

A metodologia constitui-se de um processo contínuo, pois ela contempla a prospecção científica e tecnológica da espécie Aloe vera. Na prospecção científica foram analisados os dados da base científica Scopus, que é a maior base de dados de resumos e citações de literatura revisada por pares, com ferramentas bibliométricas para acompanhar, analisar e visualizar a pesquisa. A Scopus, conforme aponta a Coordenação de Aperfeiçoamento de Pessoal de Nível Superior (CAPES), contém mais de 22.000 títulos de mais de 5.000 editores em todo o mundo, abrangendo as áreas de Ciência, Tecnologia, Medicina, Ciências Sociais e Artes e Humanidades (CAPES, 2016). Na recuperação das informações científicas, utilizou-se a palavra-chave Aloe vera, sendo considerados os artigos que apresentaram esse termo no título e/ou resumo. Os artigos encontrados foram quantificados e analisados sob diferentes aspectos, a saber: distribuição de artigos científicos por países, por ano e por área de conhecimento. 
Enquanto na prospecção tecnológica foram analisados os dados da base WIPO. De forma análoga, na prospecção tecnológica foram recuperados documentos de patentes utilizando a palavra-chave Aloe vera, sendo considerados os documentos que apresentaram esse termo no título e/ou resumo. Os documentos de patentes encontrados foram quantificados e para melhor análise foram distribuídos em patentes depositadas por países, por ano e por Classificação Internacional de Patentes (CIP).

Os dados foram organizados em gráficos e tabelas para melhor exposição dos dados. Para isso, foram utilizadas planilhas do Microsoft Word e Excel versão 2016. A pesquisa bibliográfica foi desenvolvida baseada em materiais já publicados constituídos principalmente de artigos científicos, monografias, dissertações, teses, livros e sites especializados. Os documentos bibliográficos foram selecionados de acordo com a relação com o objeto de estudo e analisados para a contextualização teórica do problema, revelando contribuições anteriores para o este estudo.

\section{Resultados e Discussão}

Os artigos científicos foram analisados quanto aos países, aos anos de publicação e às áreas de conhecimentos. De acordo com os dados da Figura 1, os países que apresentaram a maior produção de artigos científicos são a Índia, com cerca de 2.936 artigos científicos publicados; seguida dos Estados Unidos, com 1.468 artigos científicos; a China e o Irã, com 1.155 e 794 artigos publicados, respectivamente. O Brasil fica na quinta colocação com apenas 511 artigos científicos publicados.

Figura 1 - Produção de artigos científicos da espécie Aloe vera por países

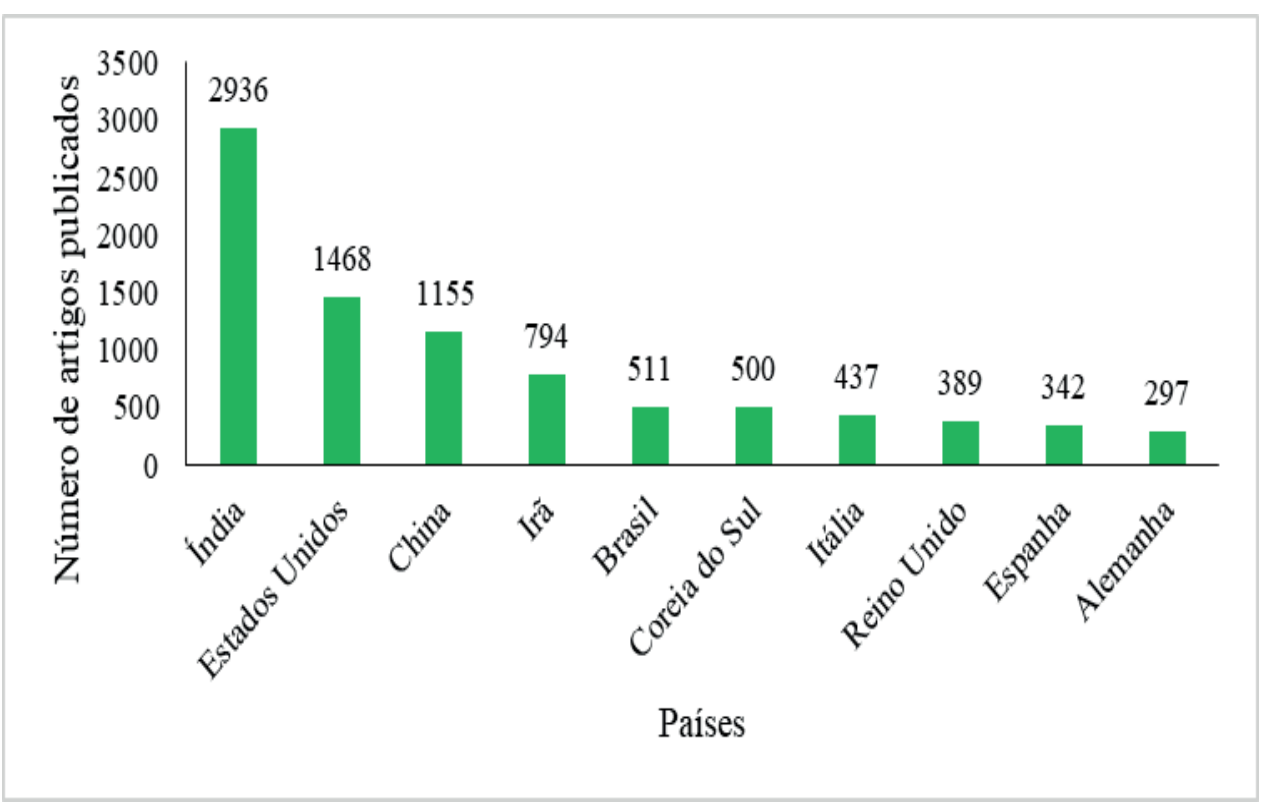

Fonte: Elaborada pelos autores deste artigo (2019)

De acordo com o exposto, percebe-se a liderança da Índia nas publicações científicas da espécie Aloe vera. O país asiático segue uma trajetória de crescimento em suas pesquisas científicas, pois parte desse sucesso indiano vem dos altos investimentos em inovação e da participação da iniciativa privada no desenvolvimento de tecnologias envolvendo a espécie Aloe 
vera. A Índia tem aumentado significativamente seu potencial mundial, o que a faz possuir uma potencialidade capaz de transformar o seu conhecimento científico em produtos. Como um país em pleno desenvolvimento, à medida que a Índia desenvolve sua iniciativa científica, diversifica a sua base de pesquisa e expande suas redes colaborativas, a nação está bem posicionada para se tornar uma das líderes da ciência no mundo. No caso, o país se destaca em pesquisa de fármacos, química, engenharia química e ciência dos materiais havendo um grande interesse daquele país pelas pesquisas sobre a Aloe vera.

Os Estados Unidos, na segunda colocação, apresentam notoriedade nessa área de pesquisa. A inovação tecnológica no país é muito forte, haja vista que consegue investir fortemente no desenvolvimento de tecnologias envolvendo a Aloe vera, seja no desenvolvimento de fármacos e derivados como na produção de produtos cosméticos. O país é responsável também por grandes investimentos em pesquisa e desenvolvimento de novos produtos em outros países. Já o Brasil, apesar de ser considerado um dos países mais importantes na pesquisa em produtos naturais, apresentou um número pouco expressivo, dada sua relevância nessa área. Ocupando a quinta posição no ranking, o país também possui produtos à base de Aloe vera.

A Figura 2 retrata a trajetória anual da publicação de artigos nos últimos dez anos. Assim, percebe-se que o ano que mais houve publicações foi em 2016 com 1.710 artigos científicos, seguido de 2015 e de 2014, com 1.472 e 1.350 artigos publicados, respectivamente.

Figura 2 - Distribuição de artigos da espécie Aloe vera por ano

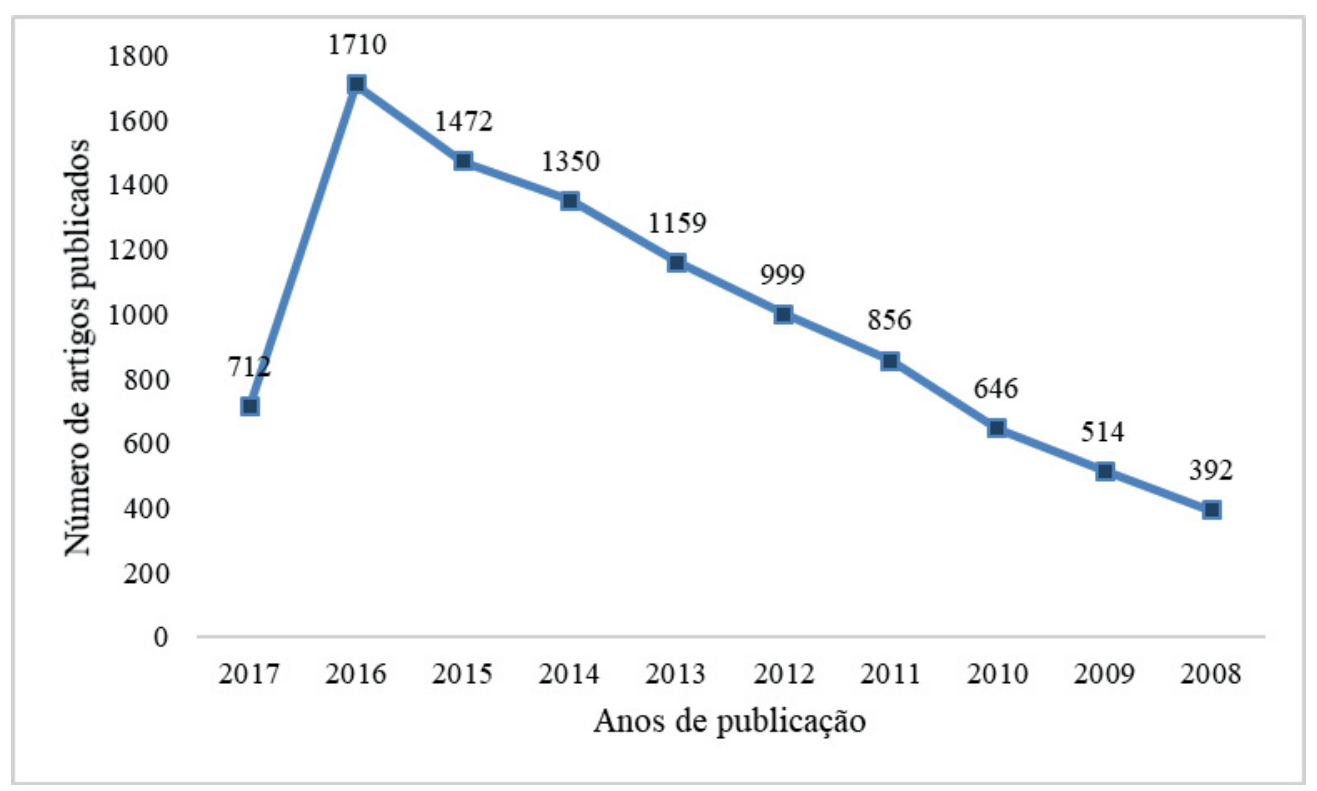

Fonte: Elaborada pelos autores deste artigo (2019)

Observa-se uma tendência na taxa de crescimento de artigos publicados ao longo de dez anos. Há um crescimento gradual em relação aos números de artigos, atingindo seu ápice no ano de 2016. Essa tendência permite concluir que o interesse científico na espécie estudada vem crescendo cada vez mais, o que comprova a importância dessa planta, sendo que ela possui inúmeras propriedades farmacológicas, por exemplo, é antibacteriana, anti-inflamatória, cicatrizante, antioxidantes, e atua no controle da glicemia, da tuberculose, da angiogênese, da fibrose hepática e de neoplasias (SOTILLI, 2015). 
O uso e aplicação da espécie Aloe vera nas áreas da Medicina, Ciências Biológicas, Química, Farmacologia e etc. demonstram sua versatilidade e, consequentemente, sua popularidade no meio científico. Conforme pode ser visto na Figura 3, a medicina está na área mais produtiva em termos de artigos científicos envolvendo a espécie Aloe vera, com um total de 4.163. A segunda área de conhecimento mais representativa é a farmacologia e toxicologia, com 2.884 artigos publicados, em seguida a agricultura e as Ciências com 2.725 artigos.

Figura 3 - Distribuição de artigos da espécie Aloe vera por área de conhecimento

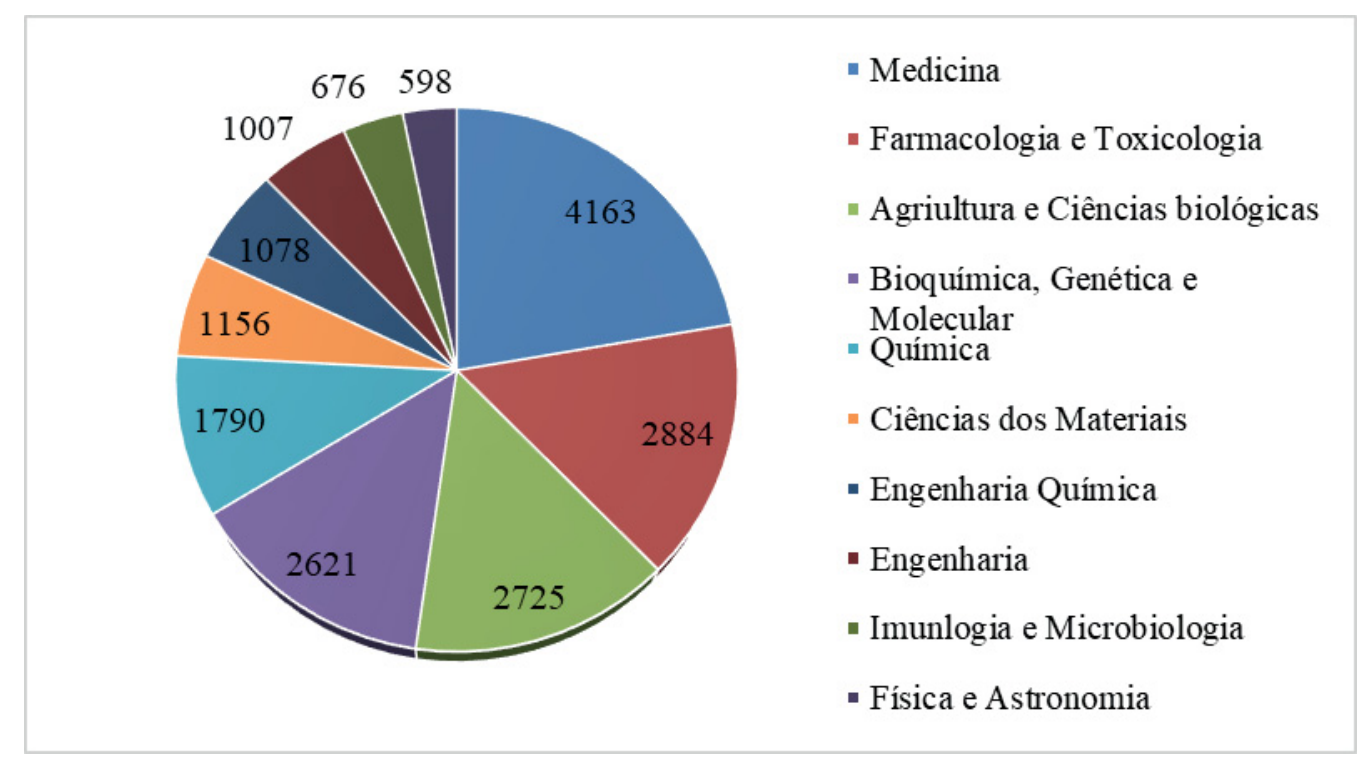

Fonte: Elaborada pelos autores deste artigo (2019)

A planta é muito utilizada com finalidade terapêutica devido às propriedades anti-inflamatórias e antibacterianas de substâncias ativas que estão concentradas tanto no gel quanto na casca das folhas de Aloe vera, que confere muitos benefícios à saúde humana (JOSEPH; RAJ; 2010; NANDAL; BHARDWAJ, 2012). Atrelada à medicina está a farmacologia e toxicologia, áreas nas quais a Aloe vera é empregada no estudo de produtos com atividades terapêticas, assim como na produção de preparações medicinais. Outras grandes áreas são a agricultura $e$ as ciências biológicas.

As análises de documentos de patentes em bases de dados tecnológicas podem ser feitas com a elaboração de indicadores tecnológicos a partir da recuperação das informações nesses sistemas de registros de patentes, com a contagem do número de patentes por países, ano, classificação CIP, entre outros. Esses documentos de patentes possuem diferentes sistemas de classificação padronizados que as classificam de acordo com o seu conteúdo tecnológico (SCOPEL; GREGOLIN; FARIA, 2013).

Os Estados Unidos se configuram como o maior detentor de depósito de patentes, com 369 depósitos registrados. Em seguida, a República da Coreia surge como o segundo maior número de depósito com cerca de 210 patentes depositadas. Outros países, como o PCT, a China e o EPO também aparecem no ranking (Figura 4). 
Figura 4 - Distribuição de patentes da espécie Aloe vera por países

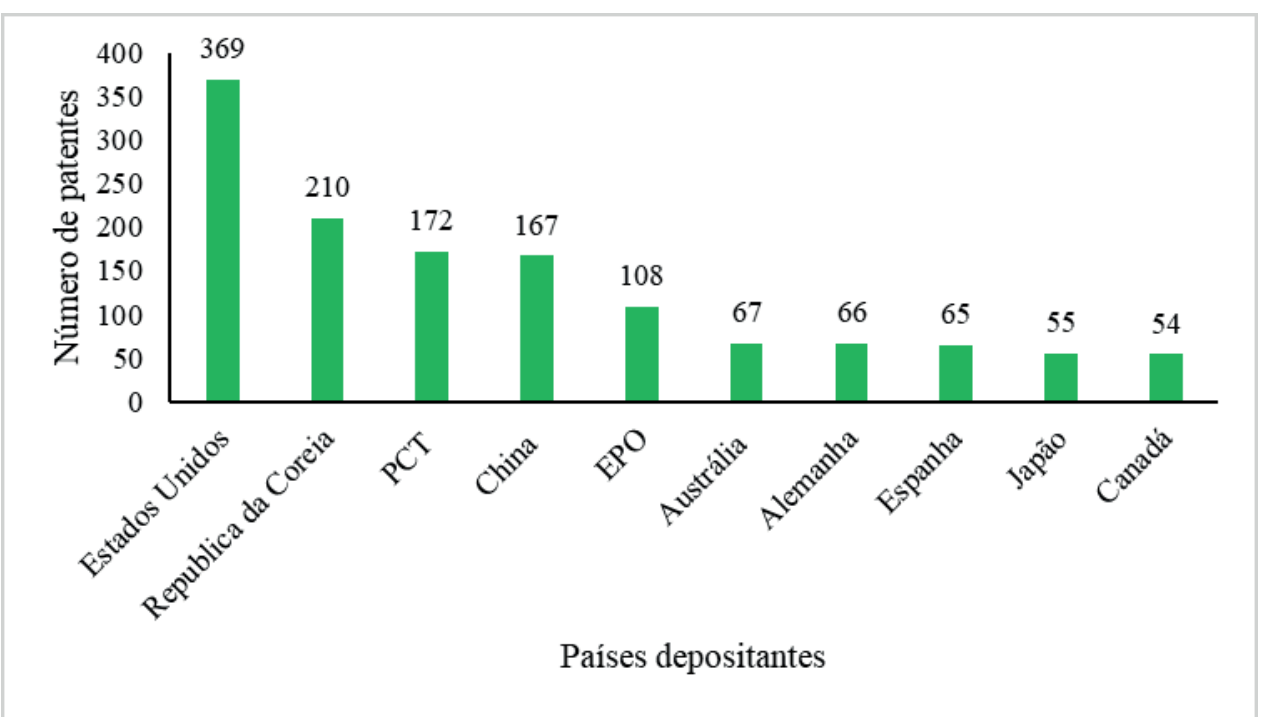

Fonte: Elaborada pelos autores deste artigo (2019)

Os Estados Unidos têm sido referência mundial no depósito de patentes de diversos produtos que vão desde a farmacologia até as patentes verdes. A Aloe vera, muito popular no país, apresenta um número bastante significativo de patentes depositadas. Esse país apresenta forte investimento por parte do governo em projetos de ciência, além de fazer cooperações e parcerias entre universidades e empresas privadas visando a fomentar o cenário da proteção intelectual e da inovação. A República da Coreia, o PCT e a China também apresentaram resultados significativos, o que evidencia que investem fortemente em pesquisas tecnológicas com a espécie em questão, seja no desenvolvimento de produtos cosméticos de cunho alimentício ou farmacológico.

O Brasil não aparece no ranking dos dez países que mais desenvolvem tecnologias com a espécie. De acordo com os dados obtidos na base de dados Scopus, o Brasil apresenta 511 artigos científicos publicados envolvendo a Aloe vera. Em relação à produção tecnológica, nosso país apresenta um número extremamente baixo, levando em consideração sua notável produção científica. Isso demonstra que o Brasil não consegue transformar o conhecimento científico produzido em tecnologias.

Ao observar a trajetória anual dos depósitos de patentes, percebe-se que o ano que mais houve depósito foi o ano de 2014, com 99 patentes depositadas, seguido dos anos de 2013 e 2006, com 94 e 84, respectivamente. Não foi encontrada nenhuma patente no ano de 2017 (Figura 5). 
Figura 5 - Número de patentes da espécie Aloe vera por ano de depósito

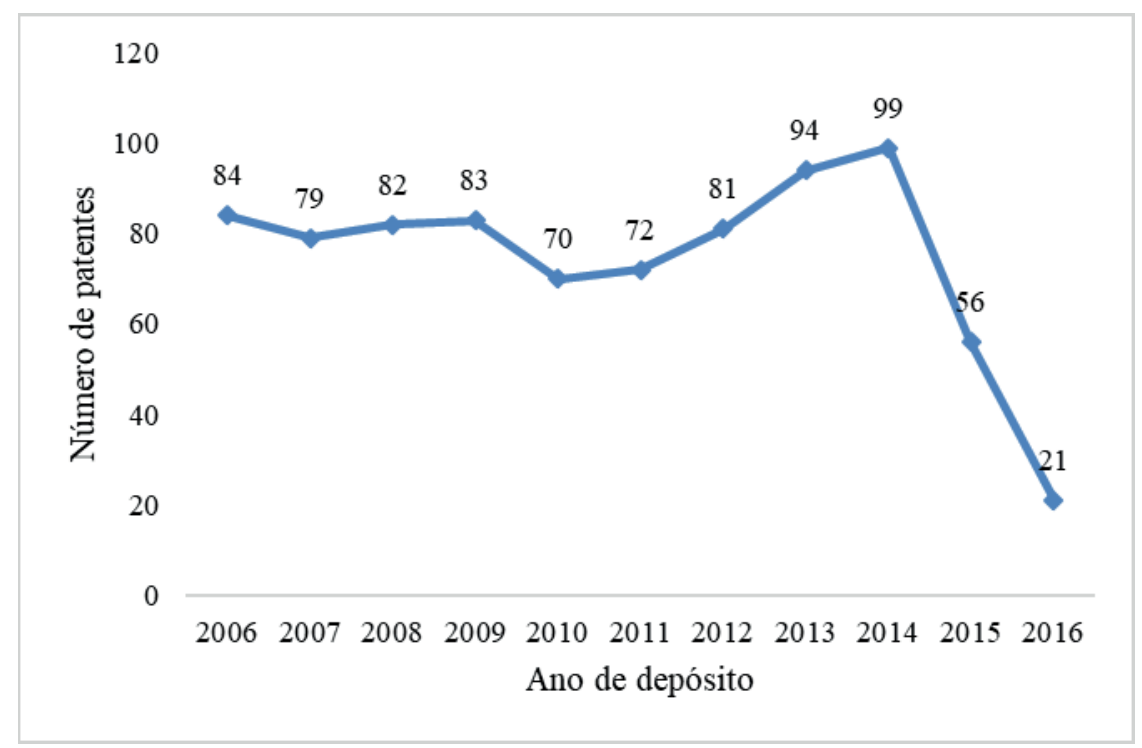

Fonte: Elaborada pelos autores deste artigo (2019)

Analisando a Figura 5, constata-se a trajetória da produção tecnológica por meio de depósitos de patentes. Percebe-se um crescimento no número de patentes depositadas nos últimos dez anos, o que indica que o interesse tecnológico da Aloe vera vem crescendo substancialmente, até o ano de 2014. A partir de então, a produção tecnológica decresce, até chegar ao mínimo, com 21 patentes depositadas no ano de 2016. Em 2017, não foi registrada nenhuma patente depositada até o presente momento.

Quanto às classificações por CIP das patentes depositadas, observou-se que a subclasse A61K apresentou o maior número de patentes depositadas, 1.126. Em seguida, estão as subclasses A61Q e A61P, com 447 e 294, respectivamente (Figura 6).

Figura 6 - Distribuição de patentes da espécie Aloe vera por CIP

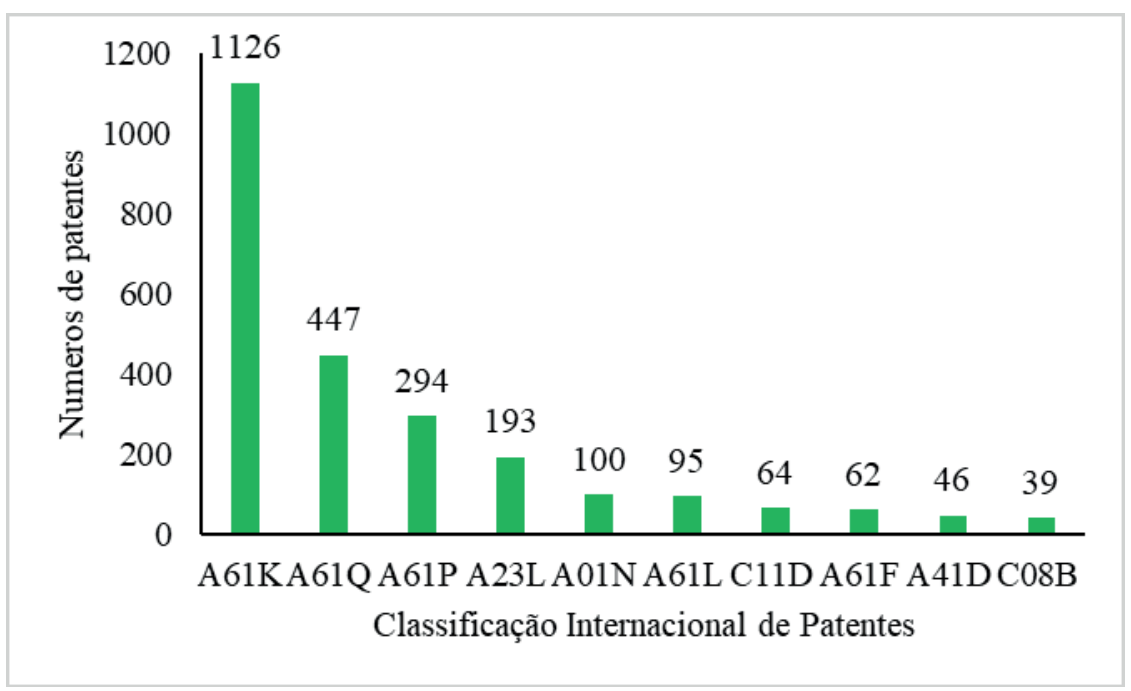

Fonte: Elaborada pelos autores deste artigo (2019)

Percebe-se que houve as classes $\mathrm{A}$, que tratam de patentes relacionadas a necessidades humanas, e a classe $\mathrm{C}$, que trata de Química e de Metalurgia. A subclasse em que houve mais classificações foi a $\mathrm{A} 61 \mathrm{~K}$, que se refere a patentes categorizadas com preparações para finalidades 
médicas, odontológicas ou de toalete. Essa subclasse abrange medicamentos ou outras composições biológicas capazes de prevenir, aliviar, tratar ou curar condições anormais ou patológicas de corpos vivos por meios como a destruição de um organismo parasita ou a limitação do efeito da doença ou do estado anormal alterando quimicamente a fisiologia do hospedeiro ou parasita $e$ composições para tratamentos do corpo, em geral destinadas a desodorizar, proteger, embelezar ou cuidar do corpo, a exemplo de cosméticos, dentifrícios, materiais para obturação de dentes.

Já a subclasse A61P abrange a atividade terapêutica de compostos químicos ou preparações medicinais. Entre as subclasses que apresentam menos patentes categorizadas são A41D e C08B. A primeira subclasse trata de artigos de usos pessoais, A41; enquanto a subseção D trata especificamente de roupas externas, trajes protetores e/ou acessórios. Já a última subclasse (C08B) abrange patentes desenvolvidas de compostos macromoleculares orgânicos, bem como a sua preparação ou o seu processamento químico; a subclasse B categoriza patentes que tratam de produtos de polissacarídeos e seus derivados.

Constatou-se que a maioria das tecnologias desenvolvidas aqui no Brasil estão categorizadas na subclasse A61K, 11 patentes depositadas, o que indica que o uso da Aloe vera se dá principalmente na indústria farmacêutica no desenvolvimento de produtos com finalidades médicas e/ou odontológicas. Outras subclasses categorizadas foram a A61L, que descreve patentes cujas tecnologias apresentam aspectos químicos, uso de materiais para ataduras ou curativos e almofadas absorventes; $\mathrm{A} 01 \mathrm{~N}$ que descreve patentes cujos produtos desenvolvidos apresentam substâncias para reduzir o efeito nocivo dos ingredientes ativos para outros organismos (bactérias, fungos etc.) e, apresentou também a subclasse $\mathrm{C} 08 \mathrm{~B}$, cujas patentes descrevem produtos de compostos orgânicos macromoleculares, bem como sua preparação ou processamento.

Segundo a Classificação Internacional de Patentes (CIP), a classe A trata de patentes cujas tecnologias são voltadas para as necessidades humanas; a subclasse 61 refere-se às patentes voltadas para higiene em geral. A subclasse $\mathrm{K}$ faz referência a patentes cujas tecnologias tratam de preparações para finalidades médicas, odontológicas ou de toalete. Essa subclasse (A61K) abrange tanto uma composição como um processo de preparo da composição ou um processo de tratamento usando essa composição e também medicamentos ou outras composições biológicas capazes de aliviar, tratar ou curar condições anormais ou patológicas de corpos vivos e composições para tratamento do corpo, em geral destinadas a desodorizar, proteger, embelezar ou cuidar do corpo (cosméticos, dentifrícios, materiais para obturação de dentes).

Entre as 13 patentes depositadas no INPI, a primeira refere-se à invenção de Banerjee, Dias e Kalathil (2014) e trata de uma fração empregada na composição de produtos utilizados para os cuidados da pele, cavidade oral e cabelo. O extrato de Aloe vera contém cerca de $60 \%$ a $95 \%$ em peso de polissacarídeos com peso molecular no intervalo de 2 a $3 \mathrm{kDa}$; sendo inferior a $5 \%$ de polifenóis, em que os polissacarídeos são hidrolisados por ácido trifluoroacético. Essa patente está classificada como A61K 8/73 (Cosméticos ou preparações similares para higiene pessoal; caracterizado pela composição; contendo compostos orgânicos macromoleculares; Polissacarídeos).

A patente de número 2 trata-se de um produto que possui propriedades antioxidantes $e$ cicatrizantes à base de Aloe barbadensis Miller, o qual é utilizado para aplicação em tratamento de feridas. Atua como cicatrizante, pois há presença de algumas substâncias, por exemplo, fenóis totais, flavonóis, taninos e saponinas. Esse conjunto de filmes apresentam os seguintes benefícios quando confrontados com os atuais produtos encontrados no mercado, como: bio- 
degradáveis, aderente, absorvível pela pele e atóxicos; podem ser umedecidos; não necessitam de curativos secundários e possuem baixo custo sendo classificada como pertencente classe A61K 36/886 - Preparações medicinais que contém materiais de constituição indeterminadas derivados de algas, líquens, fungos, ou plantas, como por exemplo, medicamentos tradicionais, base de ervas, magnoliophyta (angiospermas); liliopsida (monocotiledôneas), aloaceae (família do Aloe) (BACH; LOPES, 2007).

A patente de número 3 refere-se a um invento desenvolvido por Danhof (2011), pesquisador da North Texas Medical Associates (US), e trata-se de uma composição farmacêutica incluindo uma combinação de formulações de derivados de Aloe vera para o tratamento da Síndrome de Imunodeficiência Adquirida (AIDS) ou infecção por HIV exposto no documento. A composição inclui um injetável estéril de extrato polymannan, Raidox (antraquinonas e os seus derivados de Aloe diacetil), pó seco por congelação de Aloe vera, suco de Aloe vera e gel de Aloe. Além disso, contém um ou mais suplementos alimentares que envolvem os ácidos graxos, proteínas, sais minerais e metais, vitaminas, sais, aminoácidos, e outros excipientes aceitáveis pela indústria farmacêutica, podendo também ser utilizado para neutralizar a diarreia crônica, distúrbios digestivos, e perda de peso analisada em alguns pacientes ao longo do tratamento. Essa patente, assim como a anterior, também é classificada com a CIP A61K 36/886.

Outra patente depositada no INPI foi a de Gontijo et al. (2011) com pesquisadores da Universidade Federal de Minas Gerais (UFMG), a invenção apresenta composições farmacêuticas à base de Aloe vera e alginato que são capazes de conservar as células viáveis no interior de sua matriz por um período de 30 dias, sendo conveniente para vários tipos celulares. Os fármacos que contém amoxicilina em sua composição aumentam a viabilidade de osteoblastos (células do tecido ósseo), conservando-os biologicamente ativos. Classificada como A61K 36/296 - Preparações medicinais contendo materiais de constituição indeterminadas derivados de algas, liquens, fungos ou plantas, ou seus derivados, por exemplo: medicamentos tradicionais à base de ervas, magnoliophyta (angiospermas), magnoliopsida (dicotiledôneas), berberidaceae (família da berbis).

A patente de número 5 refere-se a uma invenção desenvolvida por Millán, Segura e Gala-Garcia (2008), pesquisadores da Universidade Federal de Minas Gerais, e trata-se de uma formulação farmacêutica de Aloe vera cujo objetivo é fazer um capeamento, ou seja, um recobrimento direto da polpa dentária com o intuito de induzir o tecido pulpar e na regeneração do tecido dentário, atuando também como matriz para transporte de fármacos e/ou células. Estas formulações desenvolvidas podem existir na forma de pó, pastas, géis, cremes, suspensões, solução, comprimidos, pomadas. Suas misturas podem estar associadas ou não a outros fármacos e com a adição ou não de vitaminas, ciclodextrinas, polímeros biodegradáveis e/ou minerais. Está classificada também na subclasse A61K 36/296.

A patente de número 6 de Rosa (2006) é a tecnologia de um tônico capilar desenvolvido à base de Aloe vera destinada a cabelos sensíveis e quebradiços, com caspas e dificuldades de crescimento devido aos desgastes naturais provocados por produtos químicos. Esse produto age no tecido capilar, fazendo com que as enzimas estimulem a vitalidade dos cabelos, restituindo e repondo os aminoácidos perdidos dos fios danificados e quebradiços, recuperando a elasticidade natural, higienizando as feridas do couro cabeludo, cicatrizando-as e eliminando as caspas. Esta patente está classificada nas subclasses A61K 36/88 (medicamentos à base de ervas, magnoliophyta (angiospermas), liliopsida (monocotiledôneas). 
A patente de número 7, depositada por Queiroz (2006), é uma invenção de curativos usados no tratamento de feridas como diabéticas, úlceras, queimaduras e feridas laceradas. Esses curativos apresentam composições que contém extratos de Aloe vera associados a substâncias como o alginato de cálcio e de sódio e também ao colágeno. Suas propriedades terapêuticas são proporcionadas pela ação do meio aquoso (80-85\%), assim como pelos componentes ativos do extrato de Aloe vera. Também está classificada na subclasse A61K 36/896.

Outra patente depositada no Brasil envolvendo a espécie em questão, indicada por Queiroz (2006), trata-se de novos curativos autolíticos para o desbridamento (remoção do tecido desvitalizado) de feridas infectadas ou necrosadas. Suas propriedades estão relacionadas à ação autolítica propiciada pelo meio aquoso e por seus componentes sobre as feridas infectadas ou necrosadas. Esta patente está classificada na subclasse A61K 36/896.

A patente de número 9 depositada na base tecnológica brasileira foi a dos pesquisadores Eng et al. (2005) e se refere a uma invenção de uma luva em que suas superfícies internas possuem uma película que contém água, glicerol e um extrato botânico. É classificada como A01N 25/34 (conservação de corpos de seres humanos ou animais ou plantas ou suas partes; biocidas, desinfetantes, pesticidas; herbicidas, folhas não mencionadas em qualquer outro subgrupo deste grupo principal).

A patente de número 10, cuja tecnologia foi desenvolvida por Weerelt (2003), trata-se do uso do sumo de Aloe vera como soluto numa solução tendo como solvente hidrolatos de materiais orgânicos e inorgânicos. Esta solução é usada como na área farmacêutica, bioquímica e industrial, e contém princípios ativos ou fármacos do sumo de Aloe vera e dos hidrolatos mencionados. Está classificada também na CIP A61K 36/886.

A patente de número 11 depositada aqui no Brasil envolvendo a espécie Aloe vera foi desenvolvida por Dijk, Goedbloed e Koumans (2002) e se refere a uma composição contendo polissacarídeos negativamente carregados derivados da Aloe vera e um processo para preparar essa composição de matéria por subfracionamento do extrato da planta. Essa composição é útil como um suplemento alimentar e para uso em cuidado pessoal e em cosméticos, especialmente para prevenir uma infecção com as bactérias. Ela está classificada na subclasse C08B 37/00 (preparações de polissacarídeos não abrangidos pelos grupos e seus derivados).

A patente de número 12 é depositada e desenvolvida por Fávere et al. (2002) e está relacionada a uma composição farmacêutica contendo quitosana e Aloe vera para uso oral em humanos com o objetivo de se obter alternativas para redução de colesterol. Está classificada na subclasse A61K 31/722 (preparações medicinais contendo ingredientes ativos orgânicos, carboidratos, seus derivados, polissacarídeos, tendo mais de cinco radicais sacarídeos ligados entre si por ligações glicosídicas; seus derivados (éteres, ésteres, glicanos e quintana).

A última patente depositada foi desenvolvida por Coelho (1995) e é a invenção de uma composição com ação depilatória, preparada com uma substância que possui inúmeras propriedades regenerativas, lubrificantes, nutritivas e umectantes, tendo como demais componentes a cera de abelha, a vaselina sólida, a essência de mel e a própria Aloe vera. Está classificada na subclasse A61K 7/155. 


\section{Considerações Finais}

Obteve-se um panorama do desenvolvimento científico e tecnológico da espécie Aloe vera. Com a prospecção científica, conclui-se que o país que mais produziu ciência envolvendo a babosa foi a Índia com 2.936 artigos, depois foi os Estados Unidos. O Brasil ocupou a quinta posição no ranking. Quanto ao desenvolvimento científico ao longo dos últimos 10 anos, observou-se um crescimento gradual. Em relação às áreas de maior interesse, destacaram-se a medicina e a farmacologia e toxicologia.

Com a prospecção tecnológica realizada, conclui-se que os Estados Unidos são o país com o maior grau de inovação tecnológica envolvendo a planta em questão, enquanto o Brasil não apareceu no ranking, o que indica que o país apresenta um baixo grau de inovação tecnológica dentro da temática da Aloe vera, apontando para um fraco investimento na produção de tecnologias sobre a espécie. Quanto ao desenvolvimento tecnológico nos últimos 10 anos, a produção de tecnologias vem decrescendo substancialmente, revelando uma falta de interesse tecnológico na espécie. Quanto à CIP, a subclasse mais categorizada foi a A61K, apontando para o maior uso da Aloe vera no desenvolvimento de produtos com finalidades médicas e odontológicas.

A partir desta pesquisa foi possível perceber que foram identificadas diversas atividades com fins farmacológicos que a Aloe vera apresenta, o que corrobora com estudos que mostram o elevado potencial de suas propriedades no tratamento de doenças, especialmente quando se trata de ações cicatrizantes, antioxidantes e antimicrobianas.

\section{Agradecimentos}

Ao Grupo de Pesquisa cadastrado no Conselho Nacional de Desenvolvimento Científico e Tecnológico (CNPq) em Alimentos, Química, Agronomia e Recursos Hídricos (AQARH).

\section{Referências}

BACH, D. B.; LOPES, M. A. Estudo da viabilidade econômica do cultivo da babosa (Aloe vera L.). Ciência e Agrotecnologia, Lavras, v. 31, n. 4, p. 1.136-1.144, 2007.

BANERJEE, G.; DIAS, P. M.; KALATHIL, R. UNILEVER PLC (a company registered in England and Wales under company no. of Unilever House, 100 Victoria Embankment, London Greater London EC4Y 0DY, 41424, GB). 2014.

CAPES - COORDENAÇÃO DE APERFEIÇOAMENTO DE PESSOAL DE NÍVEL SUPERIOR. Scopus. 2016. Disponível em: https://www.periodicos.capes.gov.br/images/documents/Scopus Guia\%20de\%20refer\%C3\%AAncia\%20r\%C3\%A1pida_10.08.2016.pdf. Acesso em: 13 jan. 2019.

COELHO, D. O. Composição para cera depilatória. PI 9504105-2 A2. Classificação: A61K 7/06. 1995.

DANHOF, I. E. Tratamento de síndrome de imunodeficiência adquirida (AIDS) com propriedades antivirais de Aloe vera L. Patente. 2011.

DIJK, W. V.; GOEDBLOED, A. F.; KOUMANS, F. J. R. Composição compreendendo polissacarídeos deriváveis de Aloe vera, extrato nag-25, extrato ultrafiltrado de aloe, seus processos de preparação, suas formas de dosagens, e seus usos. PI 0215394-7 B1. Patente. 2002. 
ENG, A. H. et al. Luva com revestimento agradável ao tato e método de fabricação. PI 0520711-8 A2. Patente. 2005.

FÁVERE, V. T. et al. Composição farmacêutica contendo cloridrato de quitosana e Aloe vera. PI 0202748-8 A2. Patente. 2002.

FREITAS, V. S. I.; RODRIGUES, R. A. F.; GASPI, F. O. G. Propriedades farmacológicas da Aloe vera (L.) Burm. f. Revista Brasileira de Plantas Medicinais, [S.l.], v. 16, n. 2, p. 229-307, 2014.

GIL, A. C. Como elaborar projetos de pesquisa. 4. ed. São Paulo: Atlas, 2002.

GONTIJO, S. M. L. et al. Composições Farmacêuticas à base de Aloe vera e alginato e seus usos. Patente. 2011.

JOSEPH, B.; RAJ, S. J. Pharmacognostic and Phytochemical properties of Aloe vera linn - an overview. International Journal of Pharmaceutical Sciences Review and Research, Bangalore, v. 4, n. 2, p. 106-110, 2010.

MILLÁN, R. D. S.; SEGURA, M. E. C.; GALA-GARCIA, A. Formulação farmacêutica à base de Aloe vera para capeamento direto em polpa dentária e como matriz para transporte de fármacos e/ou células. Patente. PI 0802009-4 A2. 2008.

NANDAL, U.; BHARDWAJ, R. L. Aloe vera for human nutrition, health and cosmetic use - A review. International Research Journal of Plant Science, Chicago, v. 3, n. 3, p. 38-46, 2012.

PARENTE, L. M. L. et al. Aloe vera: características botânicas, fitoquímicas e terapêuticas Aloe vera. Arte Médica Ampliada, [S.1.], v. 33, n. 4, 2013.

PATROCÍNIO, A. F.; MANCILHA, M. Aloe vera - abordagem técnica. In: SYNTHON ESPECIALIDADES. Sorocaba/SP. 2012. Anais [...], São Paulo: 2012.

PEREIRA, S. A. et al. Prospecção científica e tecnológica do gênero Jatropha (Euphorbiaceae). Cadernos de Prospecção, Salvador, v. 8, n. 2, p. 355-364, abr.-jun. 2015.

QUEIROZ, M. M. F. Curativo tópico a base de Aloe vera (babosa). PI 0602852-7 A2. 2006.

ROSA, I. Tônico capilar natural alôe vera. PI 0604689-4 A2. A61K 36/88; A61P 17/08; A61G 5/00. Patente. 2006.

SCOPEL, F; GREGOLIN, J. A. R.; FARIA, L. I. L. Tendências Tecnológicas do uso do Sisal em Compósitos a Partir da Prospecção em Documentos de Patentes. Polímeros, [S.l.], v. 23, n. 4, p. 514-520, 2013.

SOTILLI, C. M. Utilização de Aloe vera na promoção da saúde e seus riscos em potencial pelo uso Indiscriminado. 2015.

WEERELT, R. S. M. V. Aloe vera reconstituído com hidrolatos. PI 0308498-1 A61K 35/78. 2003.

WHO - WORLD HEALTH ORGANIZATION. WHO Monographs on selected medicinal plants Geneva: WHO Publications, 1999.

WIPO - WORLD INTELLECTUAL PROPERTY ORGANIZATION. About WIPO. [2019]. Disponível em: http://www.wipo.int/about-wipo/en/. Acesso em: 18 fev. 2019. 


\section{Sobre os Autores}

\section{Rayane Amorim Carvalho}

E-mail: rayane_gts8@hotmail.com

Licenciada em Ciências Agrárias pelo Instituto Federal de Educação, Ciência e Tecnologia do Maranhão - Campus Codó (IFMA).

Endereço profissional: Povoado Poraquê, s/n, Zona Rural, Codó, MA. CEP: 65400-000.

\section{Antonio Marcos da Conceição Lima}

E-mail: zootec21@gmail.com

Licenciado em Química pelo Instituto Federal de Educação, Ciência e Tecnologia do Maranhão - Campus Codó (IFMA).

Endereço profissional: Povoado Poraquê, s/n, Zona Rural, Codó, MA. CEP: 65400-000.

\section{Álvaro Itáuna Schalcher Pereira}

E-mail: alvaro.pereira@ifma.edu.br

Doutor em Engenharia e Ciência de Alimentos pela Universidade Estadual Paulista Júlio de Mesquita Filho (UNESP). Mestre em Química Analítica e Licenciado em Química, ambos pela Universidade Federal do Maranhão (UFMA).

Endereço profissional: Povoado Poraquê, s/n, Zona Rural, Codó, MA. CEP: 65400-000.

\section{Oswaldo Palma Lopes Sobrinho}

E-mail: oswaldo-palma@hotmail.com

Engenheiro Agrônomo pelo Instituto Federal de Educação, Ciência e Tecnologia do Maranhão - Campus Codó (IFMA). Doutorando e Mestre em Ciências Agrárias - Agronomia pelo Instituto Federal de Educação, Ciência e Tecnologia Goiano, Campus Rio Verde (IF Goiano).

Endereço profissional: Rodovia Sul Goiana, km 1, Zona Rural, Rio Verde, GO. CEP: 75901-970.

\section{Francisco Adelton Alves Ribeiro}

E-mail: adelton@ifma.edu.br

Doutor em Biotecnologia (RENORBIO) pela Universidade Federal do Piauí (UFPI). Mestrado em Engenharia da Computação e Sistemas pela Universidade Estadual do Maranhão (UEMA). Professor do Instituto Federal de Educação, Ciência e Tecnologia do Maranhão, Campus Codó (IFMA).

Endereço profissional: Povoado Poraquê, s/n, Zona Rural, Codó, MA. CEP: 65400-000.

\section{Sara Tamiris da Silva Costa}

E-mail: adelton@ifma.edu.br

Especialista em Hematologia Clínica e Banco de Sangue pelo Instituto Educacional Santa Catarina - Faculdade Jangada Instituto Nacional de Cursos (IESC). Bacharela em Biomedicina pela Faculdade Maurício de Nassau (UNINASSAU).

Endereço profissional: 289, Rua Des. Vasconcelos Tôrres, 111, Codó, MA. CEP: 65400-000.

\section{Terezinha Yasmin Azevedo Lopes}

E-mail: azevedoyasmin461@gmail.com

Graduanda em Bacharelado em Farmácia pela Universidade Brasil.

Endereço profissional: Rua Marechal Castelo Branco, 1332, São Pedro, Codó, MA. CEP: 65400-000. 